\title{
Aerodynamic investigation of a 3.5:1 prolate spheroid
}

\author{
G. Carbone*, G. Martinat \\ FLYING-WHALES, 13 Rue Pages, 92150, Suresnes, France
}

D. Farcy

Univ. Lille, CNRS, ONERA, Arts et Metiers ParisTech, Centrale Lille, FRE 2017 - LMFL - Laboratoire de Mécanique des fluides de Lille - Kampé de Feriet, F-59000 Lille, France

J.L. Harion

IMT Lille Douai, 764 boulevard Lahure, 59500, Douai

\begin{abstract}
Experimental measurements, steady (RANS) and unsteady (URANS) numerical simulations are performed on a 3.5:1 prolate spheroid at angles of attack from $0^{\circ}$ to $90^{\circ}$. The Reynolds number considered is $\operatorname{Re}_{L}=2.57 * 10^{6}$, corresponding to the wind tunnel conditions. CFD simulations are performed with OpenFOAM, using the $k-\omega$ SST and the Spalart-Allmaras turbulence models. Analyses of aerodynamic coefficients and surface pressure show a good agreement between CFD simulations and experimental results. The turbulent structures in the wake of the spheroid have a complex topology, strongly dependent on the angle of attack considered. Results of URANS simulations allow to identify turbulent structures that are steady and asymmetric at low angles of attack, symmetric and unsteady at higher values. The configuration at $A o A=90^{\circ}$ is the most complex with the presence of disorganized and unstable structures.
\end{abstract}

\section{Nomenclature}

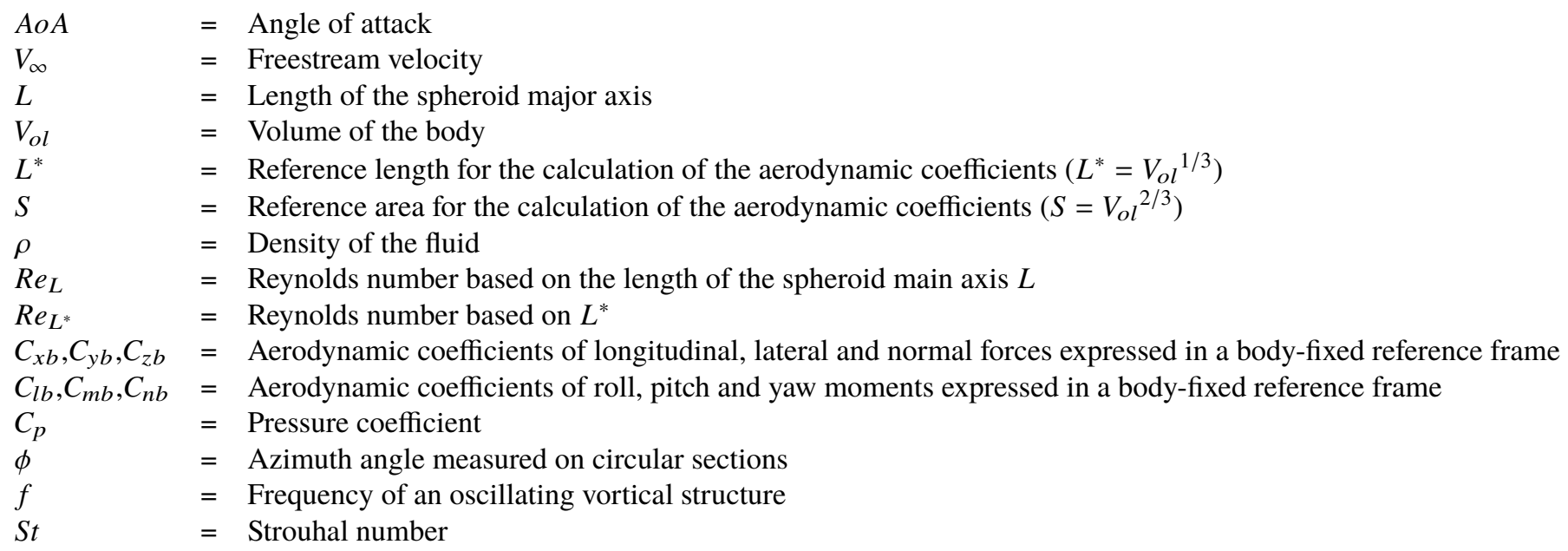

\section{Introduction}

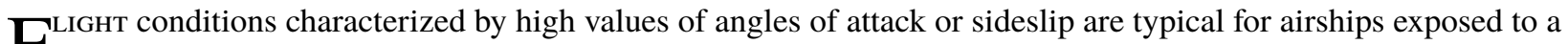
$\mathrm{F}_{\text {crosswind. The stationary flight is a natural example. The flow structures that appear behind the vehicle present }}$ turbulent wakes entrained by three-dimensional separations. The topology of these fluid structures is peculiar to bluff bodies. It is strongly influenced by the Reynolds number and by the orientation of the body with respect to the flow direction.

\footnotetext{
*PhD Student, giovanni.carbone@ flying-whales.com.
} 
Previous studies on prolate spheroids, simplified shape of airship and submarines, allowed to obtain a better understanding of the phenomena of three-dimensional separations. In contrast to two-dimensional cases, separations of three-dimensional flows are not closely related to flow reversal and the vanishing of the wall shear stress. For this reason, the prediction of separate three-dimensional flows is very challenging.

Experimental studies on a prolate spheroid (6:1 axis ratio) at a Reynolds number of $4.2 * 10^{6}$ and angles of attack of $10^{\circ}$ and $20^{\circ}$, were conducted at the Virginia Polytechnic Institute by Chesnakas and Simpson [1]. Flow separations were observed with a miniature, three-dimensional, fiber-optic LDV and through surface pressure measurements.

The same studies were conducted by Constantinescu et al. [2], through RANS and DES simulations. The mean quantities predicted by the two numerical models showed a good agreement with the experimental results obtained by Chesnakas [1] and Wetzel [3].

Direct numerical simulations were performed by El Khoury et al. [4, 5] on a 6:1 prolate spheroid at an angle of attack of $90^{\circ}$ with respect to the flow direction. The simulations were carried out at Reynolds numbers from 50 to 300 and a further study was carried out at $R e_{L}=10000$. Different wake flow phenomena have been observed, some of which are present in the wake behind a cylinder or a sphere.

A numerical study carried out on the 6:1 prolate spheroid by Jiang et al. [6] at $R e_{L}=3000$ with an angle of attack of $45^{\circ}$ showed the presence of a non-axisymmetric wake and a helical vortex as main coherent flow structure.

The flow topology observed in these studies is very different. The existence of coherent wakes, periodic instabilities and asymmetric vortical structures strongly depend on the geometry considered, and also on the Reynolds number and the angle of attack.

The aim of the present study is to describe the aerodynamics of a 3.5:1 prolate spheroid. This less elongated configuration is more representative of the hull of a modern airship and, from a geometric point of view, is in the middle between the 6:1 prolate spheroid and a sphere. The analyses are carried out at a $\operatorname{Re}_{L}=2.57 * 10^{6}$ (corresponding to $\operatorname{Re}_{L^{*}}=8.97 * 10^{5}$ according to the dimensionless notation used for airships) at angles of attack from $0^{\circ}$ to $90^{\circ}$. Aerodynamic forces, moments and surface pressure are evaluated both from experiments and CFD calculations. The numerical investigation is conducted by solving the three-dimensional incompressible Reynolds-averaged Navier-Stokes (RANS) equations with the Menter's $k-\omega$ SST [7] and the Spalart-Allmaras [8] turbulence models.

In the following sections we will present the experimental facilities and the numerical setup used for the CFD simulations. Results show a good consistency between steady-state simulations and experimental measurements. Unsteady phenomena, that occur at very high angles of attack, are studied through unsteady simulations, presented in the final section of the paper.

\section{Experimental setups}

The experimental measurements realized for these studies are carried out in the SV4 wind tunnel at ONERA Lille. The ONERA-SV4 wind tunnel is a low speed, Eiffel type, vertical wind tunnel with a $4 m$ diameter open section. The model is mounted on a rear sting (Figure1) which allows tests in a wide domain of angles of attack or sideslip.

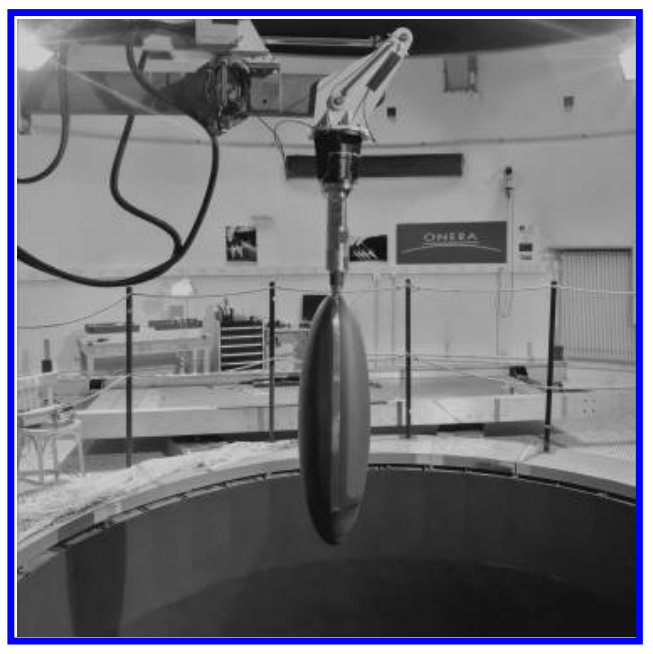

Fig. 1 Spheroid model in the ONERA-SV4 wind tunnel. 
The model is a 3.5:1 prolate spheroid. The length of the main semi-axis is $\frac{L}{2}=0.55 \mathrm{~m}$. The surface area and the reference length considered for the calculation of the aerodynamic coefficients are obtained from the spheroid volume $\left(V_{o l}=0.057 \mathrm{~m}^{3}\right)$. The respective values are $S=V_{o l}{ }^{\frac{2}{3}}=0.148 \mathrm{~m}^{2}$ and $L^{*}=V_{o l} l^{\frac{1}{3}}=0.385 m$.

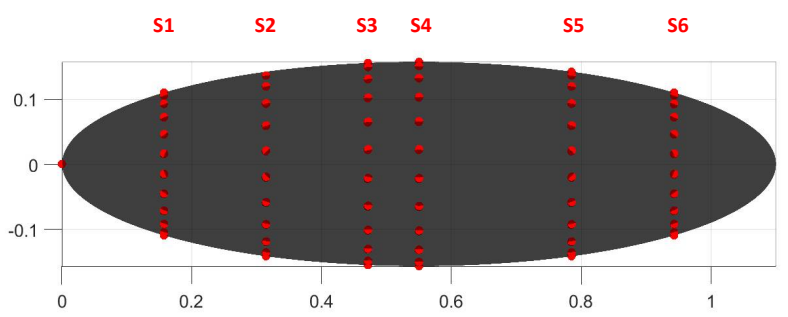

Fig. 2 Distribution of the pressure taps points on the surface of the spheroid.

The evaluation of aerodynamic characteristics is obtained through a six-component dynamometer (interface between sting and model), for measuring forces and moments and 132 pressure taps connected to five 32-channel differential pressure transducers located inside the model.

The pressure taps are distributed over 6 circular sections (Figure2) positioned at :

- $S 1$ to $x / L=0.143$

- $S 2$ to $x / L=0.286$

- $S 3$ to $x / L=0.429$

- $S 4$ to $x / L=0.50$

- $S 5$ to $x / L=0.714$

- $S 6$ to $x / L=0.857$

Where $x / L=0$ corresponds to the nose of the spheroid. The angular distribution on each section is uniform, with 22 pressure taps spaced by $\Delta \phi=16.36^{\circ}$.

\section{Numerical setups}

Numerical simulations are parameterized to reproduce the wind tunnel conditions, at $\operatorname{Re}_{L}=2.57 * 10^{6}$. The open source CFD software OpenFOAM is employed to solve the incompressible Navier-Stokes equations. The turbulence is solved whether by the $k-\omega$ SST model [7] or by the Spalart-Allmaras [8] model.

The simulation domain consists of a sphere, whose diameter is 50 times the length of the main axis of the spheroid (Figure 3 ) on whose upstream section a Dirichlet condition is imposed on the velocity and a Neumann condition on the pressure. On the outlet patch a Dirichlet condition is imposed on the pressure and a Neumann condition on the velocity. On the wall of the spheroid a non-slip condition is applied.

Different mesh sensitivity tests have been carried out. For the simulations realized with the $k-\omega$ SST model the max size of the first cells near the spheroid wall is about $y^{+}=30$ and the mean value is about $y^{+}=20$. Wall functions
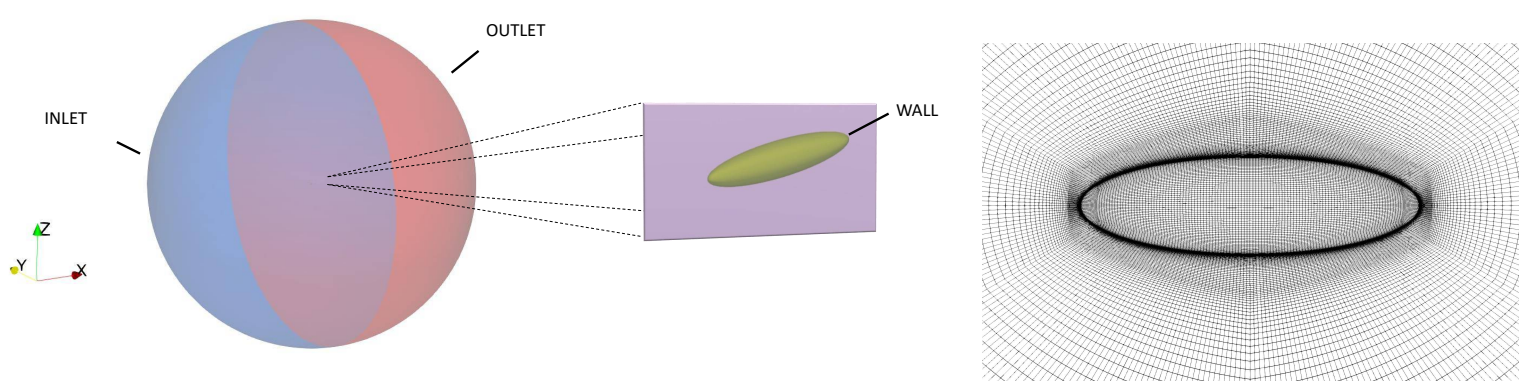

Fig. 3 Simulation domain outline and visualization of a mesh slice. 
are used to model the near wall region [9]. In the case of the simulations carried out with the Spalart-Allmaras model no wall function has been used and the maximum size of the first cell is equal or less than $y^{+}=1$. Both meshes are composed of $5.5 * 10^{6}$ hexahedral elements. Steady RANS equations are solved by the SIMPLE algorithm with a second order upwind scheme applied to the convection terms. Unsteady simulations are solved with the PIMPLE algorithm and the time derivative is calculated through a second order backward scheme.

\section{Results and discussion}

\section{A. Steady simulations and numerical results}

The studies carried out on the 3.5:1 prolate spheroid show that the topology of the flow around the body is strongly dependent on the angle of attack. At low incidences, the three-dimensional boundary layer is largely attached to the geometry and detachments are visible only on the rear zone of the spheroid. As the angle of attack increases, the adverse pressure gradient induces the detachment of the boundary layer in the leeward region with the emergence of two primary longitudinal vortices. The progressive increase of the angle of attack leads to the displacement of the boundary layer detachment towards the nose of the spheroid. As the primary vortices move away from the surface of the body, further secondary vortex structures appear in the rear area of the spheroid that join the primary structures and generate a large, coherent wake.

The phenomena just described show an extremely complex and variable fluid topology which has direct repercussions on the surface pressure distribution and on the wall shear stresses.

The comparison of experimental measurements and numerical evaluations of aerodynamic forces and moments provides a global indication of the CFD capabilities to reconstruct the fluid flows efficiently. Figure 4 represent the graphs of both experimental (the blue line and the gray zone correspond respectively to the mean value and the standard deviation obtained from experimental measurements) and numerical force and moment coefficients, represented with respect to a body-fixed reference frame.
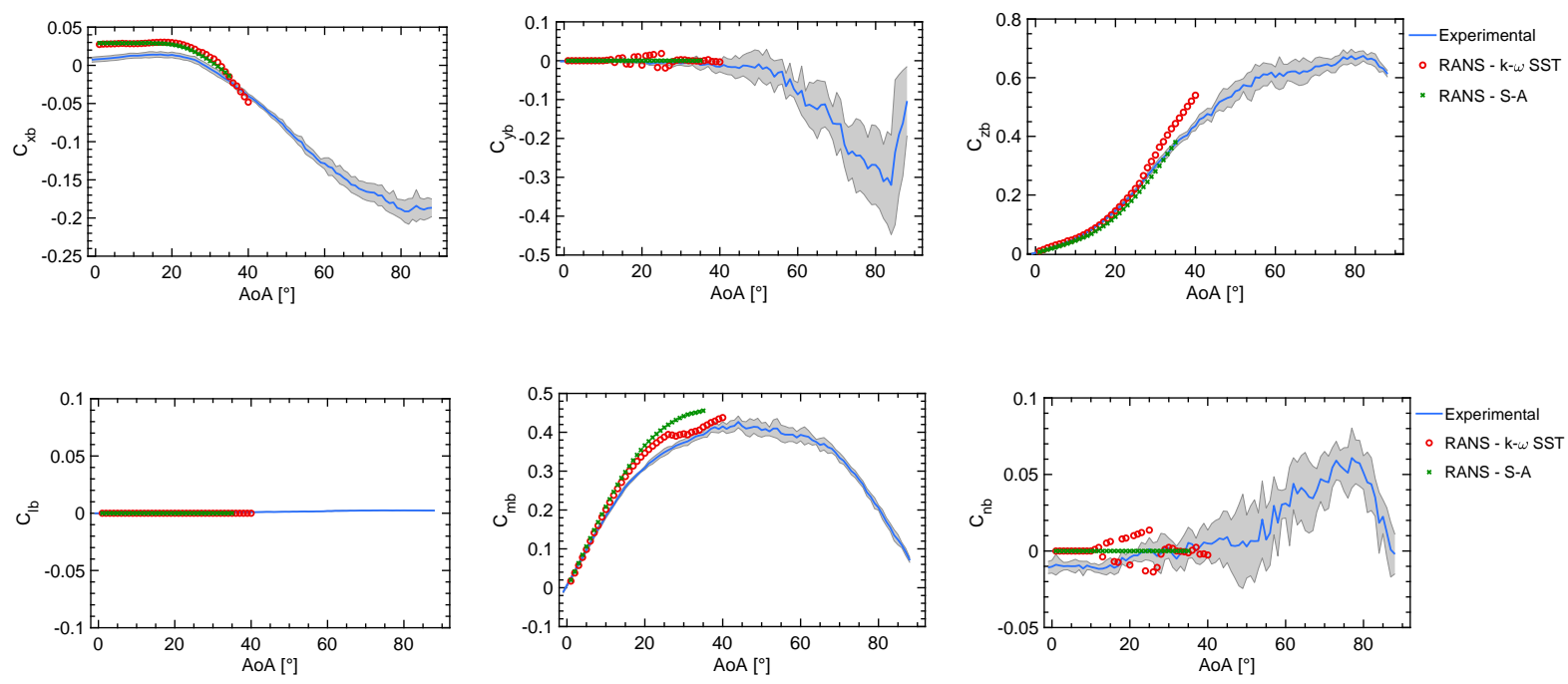

Fig. 4 Experimental and numerical aerodynamic coefficients of a 3.5:1 prolate spheroid at $\operatorname{Re}_{L}=2.57 * 10^{6}$.

Dimensionless values are obtained through the following relations:

$$
\begin{gathered}
C_{i b}=\frac{F_{i b}}{\frac{1}{2} \rho V_{\infty}^{2} S} \\
C_{j b}=\frac{M_{j b}}{\frac{1}{2} \rho V_{\infty}^{2} S L^{*}}
\end{gathered}
$$


The steady simulations realized with the $k-\omega$ SST turbulence model are carried out at angles of attack between $0^{\circ}$ to $40^{\circ}$, every degree. The simulations with the Spalart-Allmaras model, at angles of attack between $0^{\circ}$ to $35^{\circ}$, every degree.

Results obtained through the two turbulence models show a similar trend when $A o A \leq 20^{\circ}$. In this domain we can notice that the numerical simulations overestimate the longitudinal force coefficient, if compared to the experimental values. The normal force ( $\mathrm{z}$ direction) calculated by the $k-\omega \mathrm{SST}$ simulations follows the experimental values perfectly, but it is slightly underestimated by the Spalart-Allmaras model. The pitch moment obtained through the CFD simulations is consistent with the experimental values only at incidences below $10^{\circ}$, while it tends to be overestimated as the incidence increases.

These discrepancies between the numerical and the experimental results can be due to multiple factors. First, from a geometric point of view, the experimental model has a support positioned in the rear region of the spheroid. This has an influence on fluid detachments and the emergence of vortical structures, with a consequent repercussion on the values of forces and moments. Small imperfections on the surface of the experimental model can induce separations in regions that differ from those calculated by the numerical simulations. In parallel, it is well known that the closure models of RANS equations have difficulties in reproducing configurations with large separations and recirculating zones.

It should also be noted that the results calculated by the two turbulence model differ among them, showing a different topology of flows. At angles of attack between $10^{\circ}$ and $28^{\circ}$, the results of the $k-\omega$ SST model reveal asymmetrical secondary vortex structures generated in the rear section of the body. The wake resulting from the fusion of these structures with the primary ones presents stable asymmetric vortices that are randomly positioned either to the left or to the right side of the spheroid, as illustrated in Figure 5(a) Results of simulations obtained through the Spalart-Allmaras turbulence model, on the other hand, present perfectly symmetrical vortical structures (Figure 5(b)), for all angles of attack analyzed. The asymmetry previously described can also be observed from the graphs of the lateral force and yaw moment ( $C y b$ and $C n b$ of Figure 4 ).

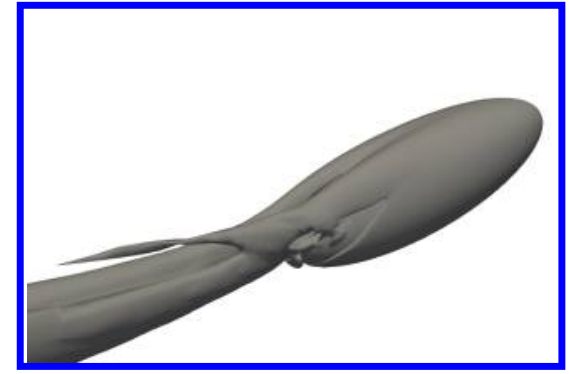

(a) $k-\omega$ SST model

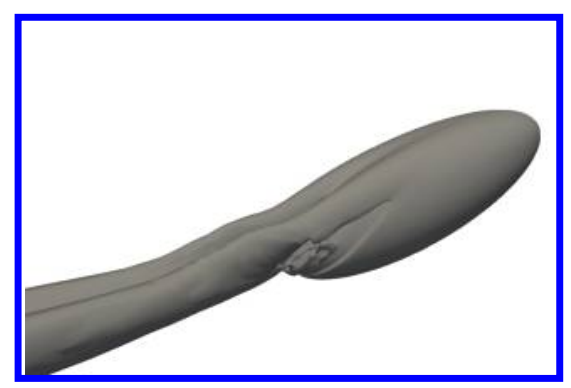

(b) Spalart-Allmaras model

Fig. 5 Visualizations of the vortical structures in the wake of the prolate spheroid at $A o A=20^{\circ}$. The structures are shown by iso-surfaces of Q-criterion.

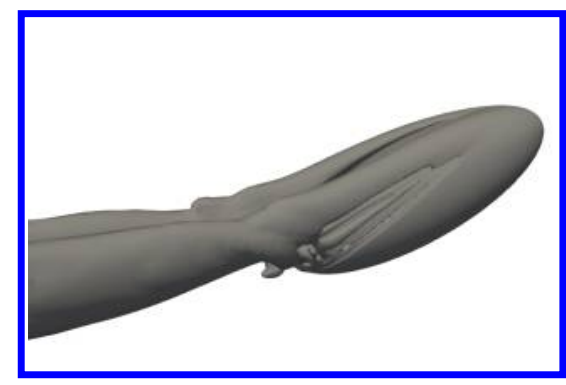

(a) $k-\omega$ SST model

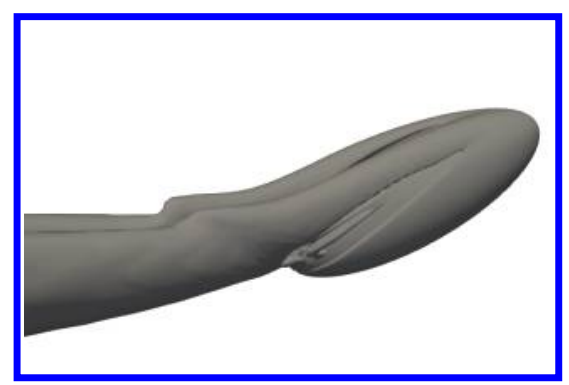

(b) Spalart-Allmaras model

Fig. 6 Visualizations of the vortical structures in the wake of the prolate spheroid at $A o A=35^{\circ}$. 
At angles of attack between $29^{\circ}$ to $40^{\circ}$ a symmetrical wake is calculated by both turbulence models (Figure 6).

The experimental values show that when $A o A \geq 40^{\circ}$, the lateral force and the yaw moment are amplified. This suggests the re-establishment of a flow asymmetry and the presence of fluid unsteadiness detectable by the amplification of the standard deviation, with respect to the mean value of the coefficients.

By analyzing the local distribution of the pressure on the surface of the body, more detailed information about the topology of the flow is deduced, identifying traces of the large primary vortices and of the secondary vortex structures.

Figure 7 shows the circumferential pressure distribution over six sections of the spheroid (Figure 2) at $A o A=35^{\circ}$. The value at $\phi=0^{\circ}$ corresponds to the windward point on the longitudinal symmetry plane of the body. Moving counterclockwise we find the point at $\phi=180^{\circ}$ on the same symmetry plane but in the leeward region.
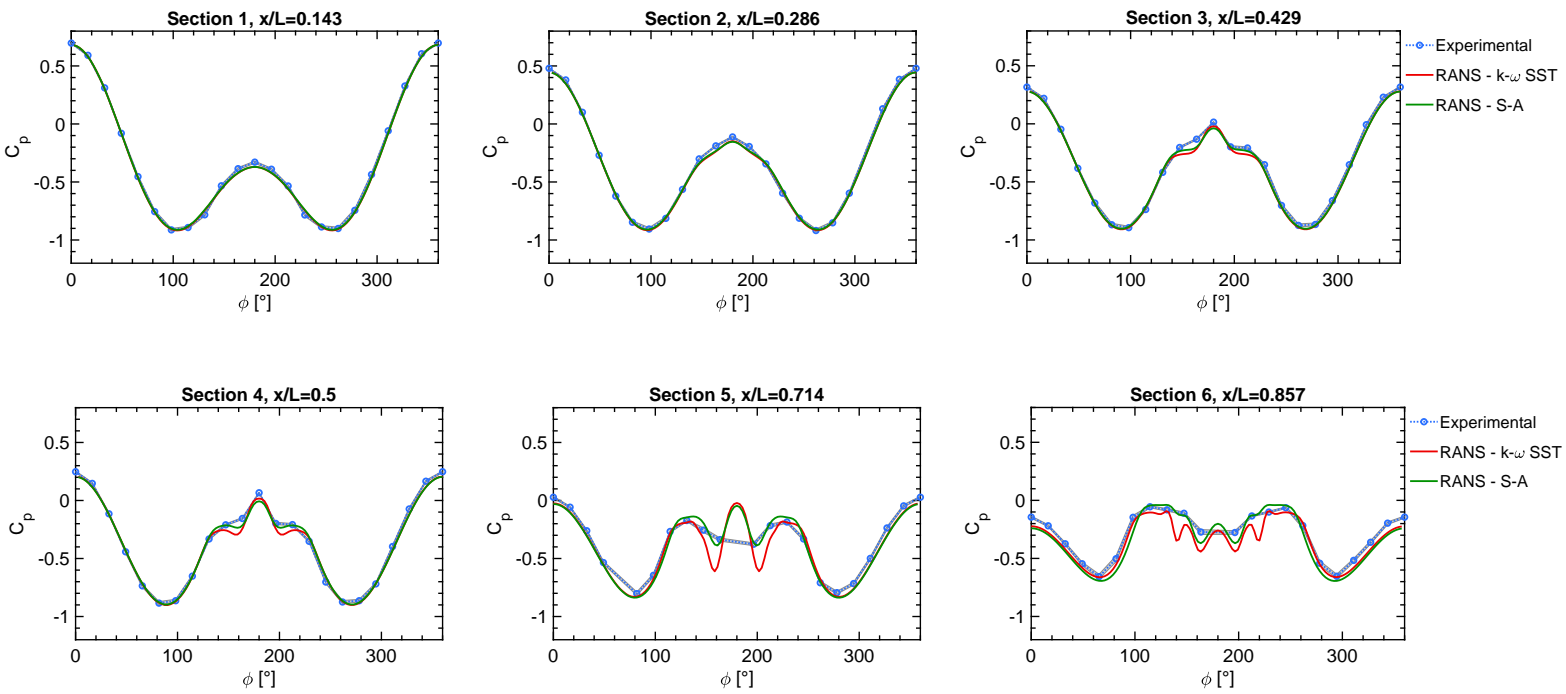

Fig. 7 Experimental and numerical pressure coefficients at $A o A=35^{\circ}, \operatorname{Re}_{L}=2.57 * 10^{6}$.

The graphs show a good consistency of the numerical profiles with the experimental ones. In the first two sections the flow is attached to the surface of the body. The numerical and experimental pressure profiles follow the same trend. Moving away from these sections, toward the tail of the spheroid, where the detachment of the flow appears, we notice some differences between the solutions obtained by the two turbulence models. The depression peaks around $\phi=160^{\circ}$ and $\phi=200^{\circ}$ (therefore in leeward area of the spheroid) calculated by the $k-\omega$ SST model are much more pronounced than those of the Spalart-Allmaras model. These differences are explained by the different near wall resolution of the boundary layer obtained with the two meshes and the two turbulence models, that induces a detachment of the flow on different positions of the body, affecting the development of the vortex structures.

Nevertheless, it is evident that RANS simulations with both turbulence models provide a very satisfactory result for the challenging configuration of the prolate spheroid.

\section{B. Unsteady simulations}

This section is dedicated to the analysis of the results of unsteady simulations, carried out with the $k-\omega$ SST turbulence model on $15^{\circ}, 35^{\circ}$ and $90^{\circ}$ angles of attack configurations. The objective of these studies is to identify the nature of unsteady phenomena or fluid-dynamic instabilities typical of bluff body wakes.

Firstly, the unsteady results of the $A o A=15^{\circ}$ simulation are presented. Steady-state results suggested the presence of an asymmetric wake for the configurations at $10^{\circ} \leq A o A \leq 28^{\circ}$. In the graphs of Figure 8 are illustrated the values of the force coefficients as a function of time ( $t^{*}$ is a dimensionless time calculated as $t V_{\infty} / L$ ). The force coefficients initially exhibit an unsteady behavior, which stabilizes and becomes constant for $t^{*}>45$. It is interesting to note that the values of $C_{y b}$ are different from 0 , indicating a flow asymmetry. The same value, but of opposite sign, was obtained from the steady simulation. These results prove that the vortex asymmetry is bi-stable, reaching an equilibrium position on the left or right side of the body randomly. 

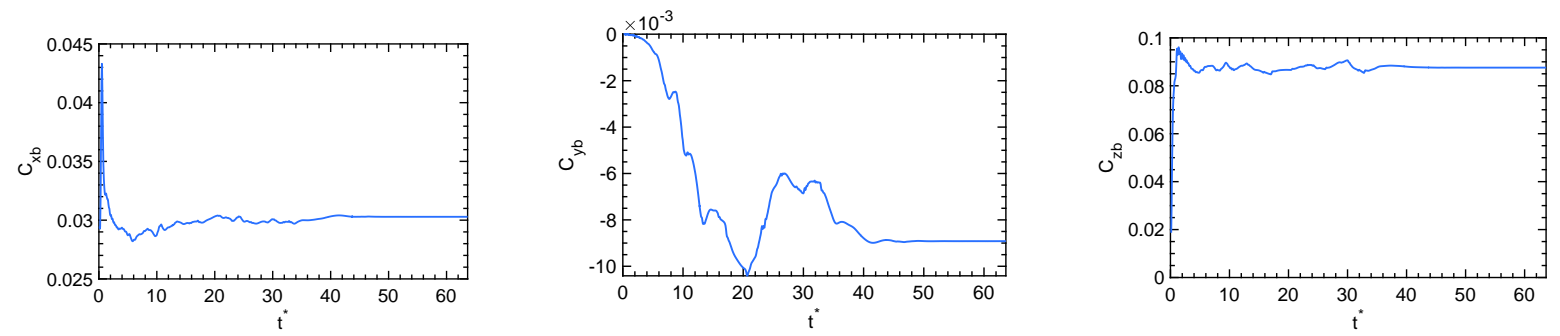

Fig. 8 Force coefficients of the unsteady simulations at $A o A=15^{\circ}, \operatorname{Re}_{L}=2.57 * 10^{6}$.

The second unsteady simulation is performed on the $A o A=35^{\circ}$ configuration. The results of the force coefficients are shown in Figure 9. Unlike the previous case, after a brief transient phase, the coefficients $C_{x b}$ and $C_{z b}$ show a periodic and oscillatory behavior. The lateral force coefficient, on the other hand, is stable and centered on 0 . From this preliminary analysis it can be deduced that, in this case, the wake of the body is symmetrical (as already shown by the steady simulation, Figure 6(a) and subject to fluctuations in the longitudinal and normal direction to the body.
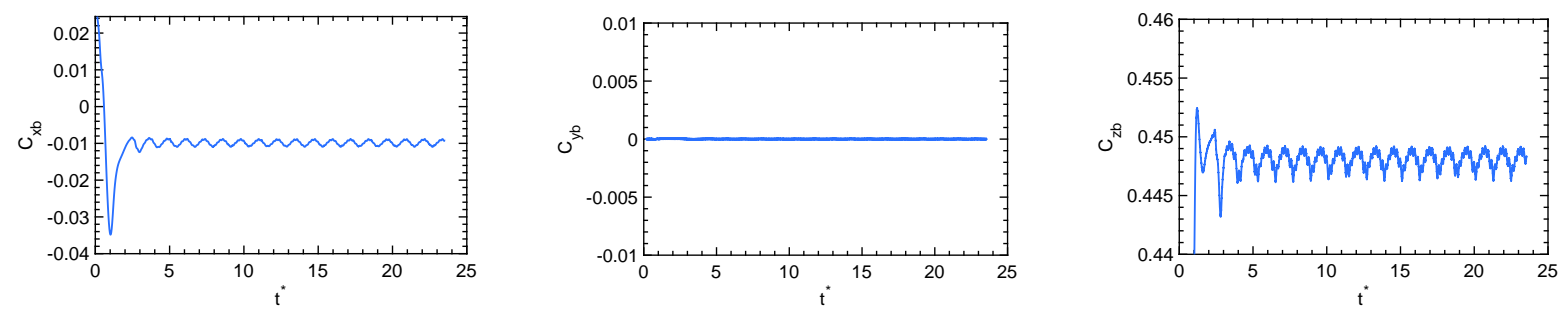

Fig. 9 Force coefficients of the unsteady simulations at $A o A=35^{\circ}, R e_{L}=2.57 * 10^{6}$.

Carrying out a frequency analysis of the signals shown in Figure 9 and defining the Strouhal number as:

$$
S t=\frac{f D}{V_{\infty}}
$$

in which the characteristic length $D$ corresponds to the maximum diameter of the medial section of the spheroid $\left(D=\frac{L}{3.5}\right)$, two frequencies are measured at $S t=0.233$ and at $S t=0.466$. The second value, corresponding to a higher frequency, is associated with the emission of vortical hairpin structures from the rear region of the body (Figure 10). The first value, corresponding to a lower frequency, is due to the instability of the two large primary vortex, which are perturbed by the fusion with the hairpin vortexes. The intensity of the latest instability has a greater impact on aerodynamic forces.

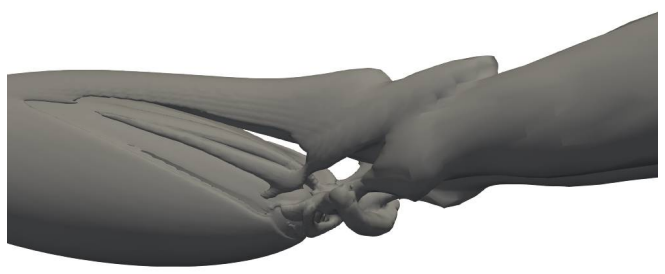

Fig. 10 Detail of the vortical structures in the wake of the prolate spheroid for the unsteady simulation at $A o A=35^{\circ}$. 
The increase of the incidence is associated with the progressive detachment of the boundary layer from the entire leeward region of the body. The vortical structures are amplified as well as their instability. The limit case corresponding to the $A o A=90^{\circ}$ configuration is described in the last part of this section. As for the two previous cases, in Figure 11 are presented the graphs of the aerodynamic coefficients as a function of time, from which a strongly unsteady and oscillatory nature of the flow is deduced. Figure 13 illustrates two snapshots of the vortical structures generated in the wake of the body. The flow appears like a Von Kármán vortex street, with a disordered and complex topology. Moreover, an asymmetry of the flow detachments between the nose and the tail of the spheroid is noticed. The asymmetry is confirmed by the $C_{x b}$ graph, where the values of the coefficient oscillate around $C_{x b}=-0.25$ and never inverts their sign.
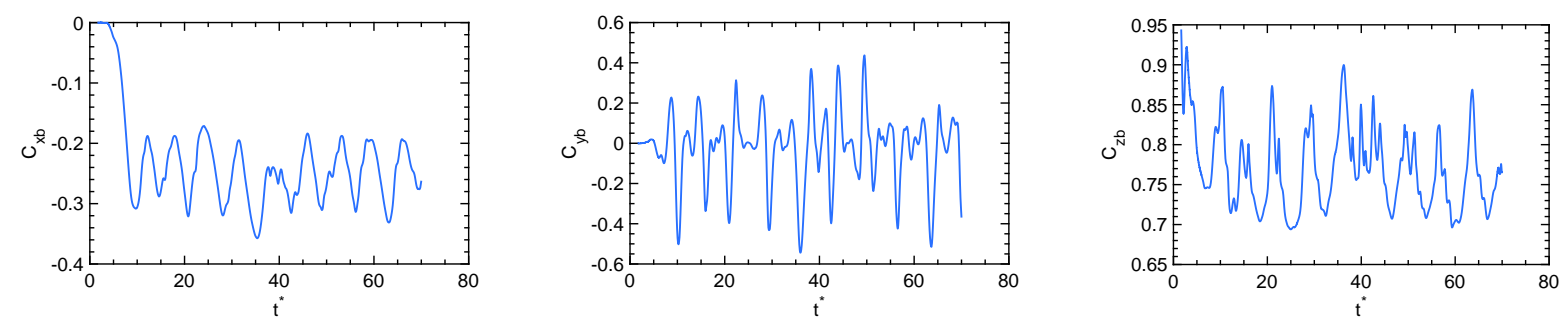

Fig. 11 Force coefficients of the unsteady simulations at $A o A=90^{\circ}, \operatorname{Re}_{L}=2.57 * 10^{6}$.

The frequency analysis is shown in Figure 12. From the graphs of $C_{x b}$ and $C_{z b}$ a predominant characteristic frequency corresponding to $S t=0.044$ is observed. More characteristic frequencies can be distinguished from the graph of the lateral force coefficient in the range between $0.044 \leq S t \leq 0.105$.
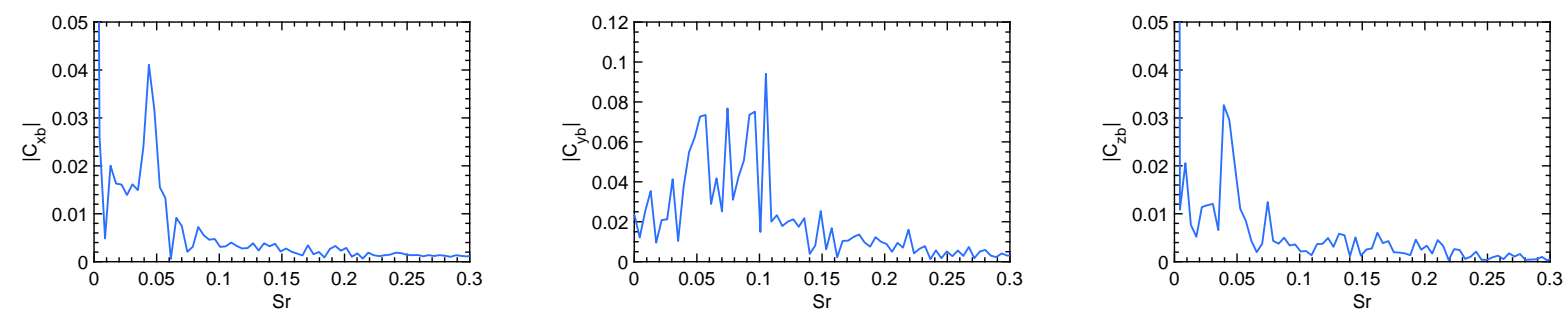

Fig. 12 Frequency analysis of the force coefficients of the unsteady simulations at $A \circ A=90^{\circ}, \operatorname{Re}_{L}=2.57 * 10^{6}$.

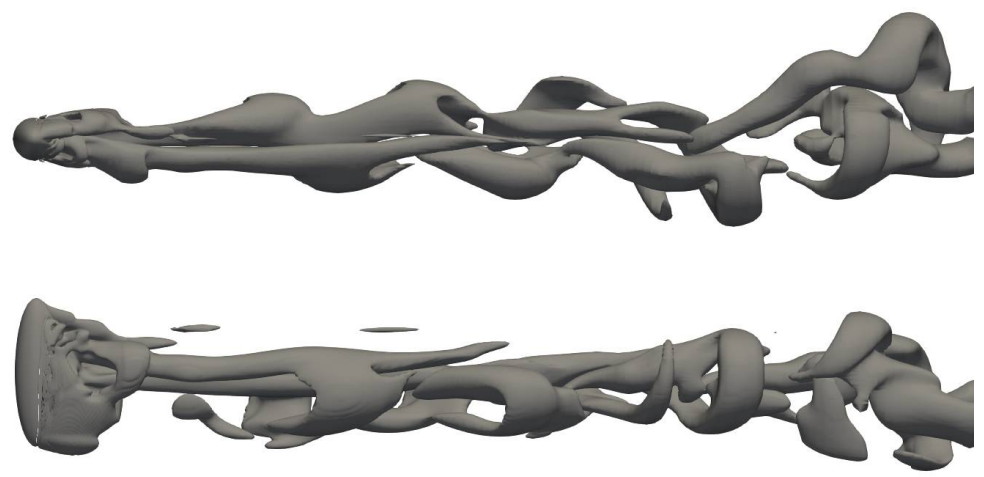

Fig. 13 Snapshots of the vortex shedding in the wake of the spheroid at $A o A=90^{\circ}$. 
Figure 14 shows the flow structures obtained from the time-averaged results of the unsteady simulation. We can clearly distinguish two large vortical structures detaching from one extremity of the body. The rest of the spheroid is enclosed by a large recirculation zone that develops in the direction of the freestream velocity. The flow topology does not differ from that of the configurations at lower angles of attack, but recalls the same characteristics, accentuated by the stronger detachment of the boundary layer.

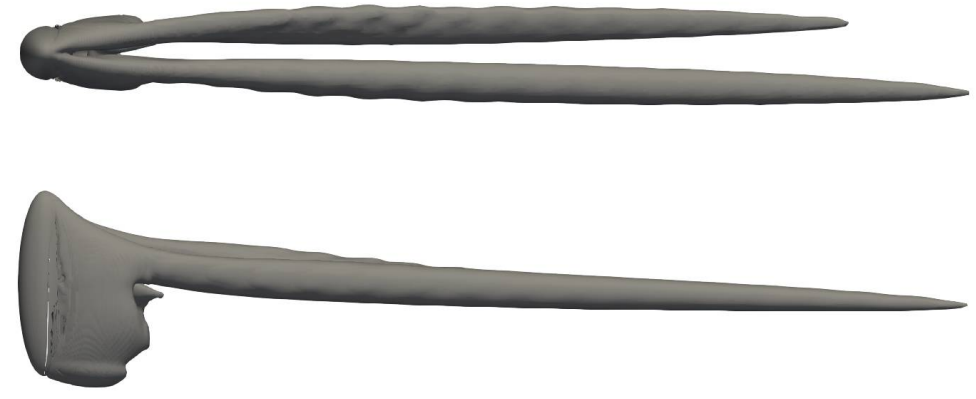

Fig. 14 Visualizations of the time averaged flow in the wake of the spheroid at $A o A=90^{\circ}$.

\section{Conclusions}

The aerodynamic description of a 3.5:1 prolate spheroid at high Reynolds number has been realized by means of wind tunnel measurements and CFD simulations. The validation of the numerical results has been achieved by comparing the force and moment coefficients, and the pressure measured experimentally and numerically. CFD simulations permitted qualitative analysis of the flow topology, visualizing vortical structures generated by the detachment and rollup of the boundary layer. The aerodynamic characteristics are strongly influenced by the angle of attack. The choice of the turbulence model, in addition to the mesh definition in the near wall region, has a significant impact on the resolution of the flows. In general, it is possible to distinguish two principal vortical structures that are generated by the nose of the body, on its leeward area. Secondary structures, of minor intensity, but unstable, are generated by further detachments of the flow from the tail of the spheroid. The wake of the body, created by the union of the primary and secondary structures, is symmetrical or asymmetrical, stable or unstable depending on the angle of attack considered. The instabilities appear and increase as the angle of attack increases, until reaching the limit condition at $A o A=90^{\circ}$. At this point the wake is strongly three-dimensional and the vortical structures detach periodically, as in the case of the wake behind cylinders or spheres.

\section{References}

[1] Chesnakas, C. J., Taylor, D., and Simpson, R. L., "Detailed investigation of the three-dimensional separation about a 6: 1 prolate spheroid," AIAA journal, Vol. 35, No. 6, 1997, pp. 990-999.

[2] Constantinescu, G., Pasinato, H., Wang, Y.-Q., and Squires, K., "Numerical investigation of flow past a prolate spheroid," 40th AIAA Aerospace Sciences Meeting \& Exhibit, 2002, p. 588.

[3] Wetzel, T. G., Simpson, R. L., and Chesnakas, C. J., "Measurement of three-dimensional crossflow separation," AIAA journal, Vol. 36, No. 4, 1998, pp. 557-564.

[4] El Khoury, G. K., Andersson, H. I., and Pettersen, B., "Crossflow past a prolate spheroid at Reynolds number of 10000," Journal of Fluid Mechanics, Vol. 659, 2010, pp. 365-374.

[5] El Khoury, G. K., Andersson, H. I., and Pettersen, B., "Wakes behind a prolate spheroid in crossflow," Journal of Fluid Mechanics, Vol. 701, 2012, pp. 98-136.

[6] Jiang, F., Andersson, H. I., Gallardo, J. P., and Okulov, V. L., "On the peculiar structure of a helical wake vortex behind an inclined prolate spheroid," Journal of Fluid Mechanics, Vol. 801, 2016, pp. 1-12.

[7] Menter, F. R., Kuntz, M., and Langtry, R., "Ten years of industrial experience with the SST turbulence model," Turbulence, heat and mass transfer, Vol. 4, No. 1, 2003, pp. 625-632. 
- [8] Spalart, and Allmaras, "A one-equation turbulence model for aerodynamic flows," 30th Aerospace Sciences Meeting and Exhibit, American Institute of Aeronautics and Astronautics, 1992. doi:10.2514/6.1992-439.

[9] Menter, F., and Esch, T., "Elements of industrial heat transfer predictions," 16th Brazilian Congress of Mechanical Engineering (COBEM), Vol. 109, sn, 2001, pp. 117-127. 